Volume 3, Issue 3, 2020, Pages 1-12

Available online at www.agricuta.edu.eg

DOI: https://dx.doi.org/10.21608/aasj.2021.54624.1047

\title{
Estimate of combining ability and correlation for yield and its components in bread wheat (Triticum aestivum L.)
}

\author{
Hammam O. Kh. , Abd-El Zaher I. N., Haridy M. H., Al-Aref Kh. A. I. \\ Agronomy Department, Faculty of Agriculture, Al-Azhar University (Assiut Branch), Assiut, Egypt
}

\begin{abstract}
The experiment was performed at the Experimental Farm of Agronomy Department, Faculty of Agricultural, Al-Azhar University (Assiut Branch), Assiut, Egypt during the three successive seasons of 2016/2017,2017/2018 and 2018/2019. Seven parents of bread wheat (Triticum aestivum, L.) of a wide diversity for some agronomic traits were selected for the study, namely Sids1 Sids12, Sids13, Shndaweel1, Giza 171, Gemmeiza 11 and Sakha 93. The parents were crossed in 2016/2017 and 2017/2018 growing seasons in a half diallel fashion. The parent and crosses were evaluated in 2018/2019. Results indicated that mean squares of genotypes, general and specific combining abilities were significant $(p \leq 0.01)$ for all traits, except number of kernels /spike. The ratio of GCA/SCA genetic components were less than unity indicating the greater amount of non- additive gene action determining the performance of all traits. The crosses (Sids 12 $\times$ Sakha 93), (Giza $171 \times$ Sakha 93), (Gemmeiza $11 \times$ Sakha 93), (Shandaweel $1 \times$ Sakha 93) and (Sids $12 \times$ Giza 171) recorded greatest mean values for grain yield /plant. Sids1 and Giza171 were considered as the best general combiners for grain yield /plant. The best crosses showed significant $(\mathrm{p} \leq 0.01)$ SCA and heterotic effects for grain yield /plant were Sids $1 \times$ Sids 13, Sids $12 \times$ Shandaweel 1 , Shandaweel $1 \times$ Sakha 93, Giza $171 \times$ Gemmeiza 11 and Giza $171 \times$ Sakha 93 so, they could consider promising hybrids in breeding programs for crop improvement.
\end{abstract}

Keywords: Triticum aestivum, combining ability, correlation, yield.

*Corresponding author: Omer K. A., 


\section{Introduction}

Wheat (Triticum aestivum L.) is one of great important food crops worldwide and in Egypt. Due to its high converting and various utilization, high nutritive value, with high crop production, it is used as major food for further one third of the global population. Improving high yielding cultivars is one of the main objectives of wheat breeding programs. Identifying the good combiners for yield and its components is of main interest for many breeders (Madic et al., 2005). For improving wheat yield, the study of the genetic structure and combining ability is of great significance for the wheat breeders. Half diallel mating is an effective strategy to assess genotypes used as parents for combining ability effects to select acceptable, parents for developing recently developed cultivars (Hayman, 1954a,b; Jinks, 1954). Successful breeding programs need to know the type of gene action and genetic system controlling the inheritance of the interest traits and the best breeding strategy to be used to improve them (Madic et al., 2005). Several researchers like Khalifa et al. (1984), Hendawy (1990), El-Shal et al. (2014), Khaled and Abd El-dayem (2014), Samier and Ismail (2015) and Rahul and Kandalkar (2018) showed that both additive and nonadditive gene actions played an equal role in the inheritance of grain yield, number of spikes /plants, number of kernels /spike and 100-kernal weight. El-Hennawy (1992), Darwish (1992), Abd El-Mageed (1995) and Mahmoud (1999) indicated that dominance and additive gene effects were significant for grain yield/plant, number of kernels /spike and 100-kernal weight. Similar results were obtained by El-Sayed et al. (2000), Hamada and Tawfeleis (2001), El- Sayed (2004), Abdel-Nour Nadya et al. (2009), Moussa (2010) and El-Awady and Wafaa (2011). Significant heterotic effects were obtained for many traits by El-Beially and ElSayed (2002), Hamada and El-Beially (2003), Megahed Eman et al. (2014) and Samier and Ismail (2015). The aim of this article was estimation both of general combining ability (GCA), specific combining ability (SCA) and heterosis for grain yield and its attributes in 21 wheat crosses and their parents.

\section{Materials and methods}

The experiments were carried out during the three growing seasons of 2016/2017, $2017 / 2018$ and 2018/2019 at the Experimental Farm of Faculty of Agriculture, Al-Azhar University, Assiut, Egypt. Seven varieties of bread wheat widely different in their agronomic characters were used as parents in this study. These varieties were Sids $1\left(\mathrm{P}_{1}\right)$ Sids12 $\left(\mathrm{P}_{2}\right)$, Sids13 $\left(\mathrm{P}_{3}\right)$ Shandaweel1 $\left(\mathrm{P}_{4}\right)$, Giza $171\left(\mathrm{P}_{5}\right)$, Gemmeiza11 $\left(\mathrm{P}_{6}\right)$ and Sakha93 $\left(\mathrm{P}_{7}\right)$ were crossed in a half diallel fashion. The description and origin of these varieties are shown in Table (1).

\subsection{Experimental layout}

In 2016/2017 season, the seven parents were sown in the field in two planting 
dates with two weeks in between to obtain enough flowers for crossing. Parents were crossed in all possible combinations excluding reciprocals to produce $21 \mathrm{~F}_{1}$ hybrids. In 2017/2018 season, parents were crossed again in to obtain more hybrid seeds ( $\mathrm{F}_{1}$ 's) for all combinations. In 2018/2019 season, the parents and their $21 \mathrm{~F}_{1}$-hybrids were sown in a randomized complete block design (RCBD) with three replications. Planting was carried out on November $20^{\text {th }}, 2018$. Plants were grown in rows, 4 $\mathrm{m}$ long and $50 \mathrm{~cm}$ apart, in single seeded hill spaced $15 \mathrm{~cm}$. Each parent and $\mathrm{F}_{1}$ hybrid were represented by three rows /plot. The agricultural practices of irrigation and fertilization were carried out as recommended for wheat production. The data were recorded on the mean of ten guarded plants /plot for both of parents and $\mathrm{F}_{1}$ hybrids. Mid parents heterosis $(\%)=\left(\mathrm{F}_{1}-\mathrm{mid}\right.$ parent/mid parent) $\mathrm{x} 100$. Better parents heterosis $(\%)=\left(\mathrm{F}_{1}-\right.$ better parent/better parent $) \times 100$. The analysis of combining ability was performed as outlined by Griffing (1956). The recorded characters were days to $50 \%$ blooming (day), plant height $(\mathrm{PH}, \mathrm{cm})$, number of spikes /plants (NS/P), spike length of (SL, cm), number of grain /spike (NG/S), 1000grain weight $(\mathrm{GW}, \mathrm{gm})$ and grain yield /plant (GY/P, gm).

Table (1): The Pedigree and origin of the seven bread wheat parental varieties.

\begin{tabular}{|l|l|c|}
\hline Parent & Pedigree & Origin \\
\hline Sids 1 & MRL/BUC/SER1 & Egypt \\
\hline Sids 12 & $\begin{array}{l}\text { BUC//7C/ALD/5/MAYA74/0N//1160 } \\
\text { Egypt/47/3/BB/GLL/4/CHAT"S"/6/MAYA/VUL - } \\
\text { //CMH74A.63014*SX.SD7096-4SD-1SD-1SD-0SD. }\end{array}$ & Egypt \\
\hline Sids 13 & $\begin{array}{l}\text { ALMAZ.19=KAUZ"S"//TSI/SNB"S"IICSBW1-0375-4AP- } \\
\text { 2AP-030AP-0APS-3AP-0APS-050AP-0AP-0SD }\end{array}$ & Egypt \\
\hline Shandaweel 1 & $\begin{array}{l}\text { Site // MO /Nac/th. Ac./3* pvn /3/Mir 10/Buc } \\
\text { Cmss93Boo567s-72Y-010M-010Y-010M-OHTY }\end{array}$ & Egypt \\
\hline Giza 171 & Sakha93/Gemmeiza9 GZ003 - 101-1GZ -1GZ - 2 GZ -0GZ & India \\
\hline Gemmeiza 11 & BOW,,s,/KVZ/7C/SERI82/3/GIZA168/SAKHA61 & Egypt \\
\hline Sakha 93 & SAKHA 92/ TR 810328: & Egypt \\
\hline
\end{tabular}

\section{Results and Discussion}

\subsection{Analysis of variance and mean performance}

The analysis of variance (Table 2) showed significant $(\mathrm{p} \leq 0.01)$ differences among genotypes for all traits, indicating a wide genetic variability in these materials and the genetic analysis could be performed. The analysis of variance in Table (2) emphasized that mean squares due to general and specific combing ability were significant $(p \leq 0.01)$ for all the studied traits, indicating that the additive and non-additive effects were involved in the inheritance of these traits. The ratio of $\sum \mathrm{gi}^{2} / \sum \mathrm{sij}^{2}$ was less than unity indicating the predominant effects of non-additive in the inheritance of these 
traits. These results are in line with those (2003), Nazir et al. (2005) and Motawea reported by Tolba (2000), Ahmadi et al. (2006).

Table (2): Mean squares of genotypes, general combining ability (GCA), specific combining ability (SCA) and their ratios for yield and its components.

\begin{tabular}{|l|l|c|c|c|c|c|c|c|}
\hline S.O.V & d.f & $\begin{array}{c}\text { Days to 50\% } \\
\text { blooming }\end{array}$ & $\begin{array}{c}\text { Plant } \\
\text { height }\end{array}$ & $\begin{array}{c}\text { Number of } \\
\text { spikes/plant }\end{array}$ & $\begin{array}{c}\text { Spike } \\
\text { length }\end{array}$ & $\begin{array}{c}\text { Number of } \\
\text { grains/spike }\end{array}$ & $\begin{array}{c}\text { 1000-grain } \\
\text { weight }\end{array}$ & $\begin{array}{c}\text { Grain } \\
\text { yield/plant }\end{array}$ \\
\hline Replicates & 2 & 1.063 & 0.210 & $8.570^{* *}$ & $7.691^{* *}$ & 0.373 & $34.84^{* *}$ & $25.61^{* *}$ \\
\hline Genotypes & 27 & $4.472^{* *}$ & $66.49^{* *}$ & $7.261^{* *}$ & $6.382^{* *}$ & $85.10^{* *}$ & $37.83^{* *}$ & $21.19^{* *}$ \\
\hline GCA & 6 & $9.826^{* *}$ & $118.6 * *$ & $7.529 * *$ & $5.474^{* *}$ & $188.9^{* *}$ & $84.66^{* *}$ & $11.19^{* *}$ \\
\hline SCA & 21 & $2.943^{* *}$ & $51.60^{* *}$ & $7.185^{* *}$ & $6.641^{* *}$ & $55.45^{* *}$ & $24.46^{* *}$ & $24.04 * *$ \\
\hline Error & 54 & 0.785 & 0.948 & 0.494 & 0.373 & 1.370 & 1.228 & 1.025 \\
\hline$\sum \mathrm{gi}^{2} / \sum \mathrm{sij}^{2}$ & & 0.40 & 0.26 & 0.12 & 0.09 & 0.38 & 0.39 & 0.05 \\
\hline
\end{tabular}

Means of parents and their $F_{1}$ hybrids are presented in (Table 3). The parental means showed wide variability for all traits. Furthermore, means of the $\mathrm{F}_{1^{-}}$ hybrids located outside the parental range indicating the presence of non-additive gene effects and/or transgressive segregation. These results agree with those reported by Fonseca et al. (1968), Afiah et al. (2000), El-Beially and ElSayed (2002), El-Borhamy (2004), Farooq et al. (2010), Khaledand Abd Eldayem (2014), Samier and Ismail (2015) and Rahul and Kandalkar (2018).

Table (3): Mean performances for all studied characters of parents and $\mathrm{F}_{1}$ crosses.

\begin{tabular}{|c|c|c|c|c|c|c|c|}
\hline $\begin{array}{|cc|}\text { Genotypes } & \text { Traits } \\
\end{array}$ & $\begin{array}{c}\text { Days to } 50 \% \\
\text { blooming }\end{array}$ & Plant height & $\begin{array}{ll} & \text { Number } \\
\text { of } & \text { spikes/plant }\end{array}$ & Spike length & $\begin{array}{c}\text { Number of } \\
\text { grains/spike }\end{array}$ & $\begin{array}{c}\text { 1000-grain } \\
\text { weight }\end{array}$ & $\begin{array}{c}\text { Grain } \\
\text { yield/plant }\end{array}$ \\
\hline Sids1 (P1) & 81.33 & 101.80 & 13.33 & 12.13 & 74.72 & 44.27 & 27.70 \\
\hline Sids12 (P2) & 83.43 & 94.30 & 10.73 & 12.93 & 79.43 & 46.43 & 27.07 \\
\hline Sids13 (P3) & 83.60 & 90.63 & 13.30 & 12.07 & 80.64 & 43.90 & 26.54 \\
\hline Shandaweel 1(P4) & 81.90 & 94.77 & 11.33 & 13.67 & 76.47 & 46.43 & 27.13 \\
\hline Giza 171 (P5) & 80.30 & 103.70 & 11.47 & 12.97 & 66.43 & 51.57 & 28.47 \\
\hline Gemmeiza (P6) & 81.49 & 105.20 & 10.00 & 12.80 & 83.57 & 42.10 & 25.83 \\
\hline Sakha 93 (P7) & 83.77 & 98.20 & 12.73 & 12.93 & 83.57 & 49.20 & 23.97 \\
\hline$P \pm$ S.E & $82.26 \pm 0.51$ & $98.37 \pm 2.05$ & $11.84 \pm 0.49$ & $12.79 \pm 0.21$ & $77.83 \pm 2.28$ & $46.27 \pm 1.23$ & $26.67 \pm 0.55$ \\
\hline $\mathrm{P} 1 \times \mathrm{P} 2$ & 80.13 & 105.60 & 14.17 & 15.13 & 81.17 & 49.23 & 30.83 \\
\hline $\mathrm{P} 1 \times \mathrm{P} 3$ & 81.50 & 104.80 & 16.50 & 13.97 & 84.57 & 48.33 & 30.30 \\
\hline $\mathrm{P} 1 \times \mathrm{P} 4$ & 81.30 & 104.90 & 14.47 & 16.53 & 79.07 & 50.93 & 30.53 \\
\hline $\mathrm{P} 1 \times \mathrm{P} 5$ & 80.47 & 108.30 & 14.30 & 14.63 & 76.93 & 53.87 & 32.07 \\
\hline $\mathrm{P} 1 \times \mathrm{P} 6$ & 79.53 & 107.20 & 13.97 & 15.30 & 84.63 & 47.17 & 30.30 \\
\hline $\mathrm{P} 1 \times \mathrm{P} 7$ & 83.53 & 105.30 & 14.33 & 15.40 & 88.50 & 53.23 & 31.47 \\
\hline $\mathrm{P} 2 \times \mathrm{P} 3$ & 83.97 & 101.20 & 14.27 & 14.67 & 85.50 & 49.93 & 29.77 \\
\hline $\mathrm{P} 2 \times \mathrm{P} 4$ & 81.03 & 100.90 & 12.10 & 16.41 & 85.53 & 51.57 & 31.77 \\
\hline $\mathrm{P} 2 \times \mathrm{P} 5$ & 81.37 & 107.30 & 11.67 & 15.63 & 83.93 & 55.30 & 32.57 \\
\hline $\mathrm{P} 2 \times \mathrm{P} 6$ & 81.83 & 109.60 & 15.30 & 15.63 & 92.60 & 49.67 & 28.67 \\
\hline $\mathrm{P} 2 \times \mathrm{P} 7$ & 81.57 & 102.40 & 16.20 & 15.37 & 86.13 & 53.20 & 35.27 \\
\hline $\mathrm{P} 3 \times \mathrm{P} 4$ & 81.70 & 107.90 & 14.53 & 16.63 & 84.40 & 49.80 & 29.60 \\
\hline $\mathrm{P} 3 \times \mathrm{P} 5$ & 82.53 & 105.50 & 14.87 & 15.87 & 81.27 & 53.60 & 31.27 \\
\hline $\mathrm{P} 3 \times \mathrm{P} 6$ & 81.87 & 107.50 & 14.50 & 14.70 & 84.57 & 48.33 & 29.87 \\
\hline $\mathrm{P} 3 \times \mathrm{P} 7$ & 82.10 & 101.10 & 13.93 & 15.27 & 90.70 & 52.33 & 28.40 \\
\hline $\mathrm{P} 4 \times \mathrm{P} 5$ & 81.53 & 105.90 & 12.67 & 16.70 & 79.43 & 54.53 & 31.40 \\
\hline $\mathrm{P} 4 \times \mathrm{P} 6$ & 82.37 & 107.50 & 13.97 & 17.27 & 87.43 & 50.57 & 29.50 \\
\hline $\mathrm{P} 4 \times \mathrm{P} 7$ & 81.43 & 100.70 & 14.20 & 16.47 & 84.10 & 52.40 & 31.80 \\
\hline $\mathrm{P} 5 \times \mathrm{P} 6$ & 73.03 & 107.80 & 14.53 & 15.63 & 88.07 & 55.00 & 32.90 \\
\hline $\mathrm{P} 5 \times \mathrm{P} 7$ & 82.30 & 106.60 & 14.40 & 15.50 & 85.87 & 55.30 & 33.83 \\
\hline $\mathrm{P} 6 \times \mathrm{P} 7$ & 82.30 & 108.40 & 14.40 & 15.50 & 85.87 & 51.90 & 23.97 \\
\hline$F_{l} \pm$ S.E & $81.30 \pm 0.47$ & $105.54 \pm 0.60$ & $14.25 \pm 0.24$ & $15.63 \pm 0.18$ & $84.77 \pm 0.82$ & $51.72 \pm 0.54$ & $30.77 \pm 0.50$ \\
\hline
\end{tabular}




\subsection{Combining ability analysis}

\subsubsection{General combining ability}

Data in Table (4) revealed that $\mathrm{P}_{5}$ (Geiza 171) has a desirable significant $(p \leq 0.01)$ gca effects for all traits, except $\mathrm{PH}, \mathrm{P}_{5}$ could be considered a good general combiner in improving days to 50\% blooming, NS/P , SL, NG/S, GW, and GY/P also, P3 has a desirable significant $(p \leq 0.01)$ gca effects for all traits, except days to $50 \%$ blooming and $\mathrm{P}_{7}$ was good combiner for all studied traits except days to $50 \%$ blooming and $\mathrm{PH}$.

\subsubsection{Specific combining ability}

Specific combining ability effects are presented in Table (4). Five crosses showed favorable negative significant SCA effects for days to $50 \%$ blooming. These crosses involved one or the two parents of negative GCA effects. However, the crosses $\mathrm{P}_{1} \times \mathrm{P}_{7}$ and $\mathrm{P}_{3} \times \mathrm{P}_{6}$ gave positive significant SCA effects, and both involved one parent of negative GCA effects. The results of days to $50 \%$ blooming indicate that the performance of this trait depended on both additive and non-additive gene effects. The SCA effects for PH showed seventeen crosses were significant $(p \leq 0.05-p \leq 0.01)$, nine of them involved $\mathrm{P}_{3}$ or $\mathrm{P}_{6}$ or both. Furthermore, four out of the nine crosses of $\mathrm{P}_{6}$ showed significant SCA effects proving that $\mathrm{P}_{6}$ can transfer favorable genes of PH. Fifteen hybrids showed positive significant $(p \leq 0.05-p \leq 0.01)$
SCA for NS/P, ten of them involved one of the parents had positive GCA, and two hybrids $\left(\mathrm{P}_{2} \times \mathrm{P}_{6}\right.$ and $\left.\mathrm{P}_{4} \times \mathrm{P}_{6}\right)$ had two parents of negative GCA effects. This indicates that NS/P controlled by additive and non-additive gene effects. The SCA effects were significant $(p \leq 0.05-p \leq 0.01)$ for $19,13,17$ and 14 hybrids for spike length, NG/S, GW, and GY/P, respectively. All these hybrids except few cases include one of their parents showed significant GCA effects. Otherwise, the cross $\mathrm{P}_{4} \times \mathrm{P}_{6}$ gave significant favorable SCA effects for all traits except GY/P despite of its parents showed negative GCA effects. Also, the cross $\mathrm{P}_{1} \times \mathrm{P}_{4}$ gave positive SCA effects for $\mathrm{SL}, \mathrm{GW}$ and GY/P, the cross $\mathrm{P}_{2} \times \mathrm{P}_{4}$ in SL, NG/S, GW and GY/P. These cases proved the presence of dominance and epistasis effects in the inheritance of these traits. It could be concluded that in the presence of non-additive the performance of the hybrids could not be predicted according to GCA effects. These results agree with the findings of Abdel-Moneam (2009), Peng et al. (2009), Sener (2009), Kundan et al. (2010), Ahmad (2010), Kumar and Gupta (2010), Padhar et al. (2010), Zaazaa et al. (2012), Khaledand Abd El-dayem (2014), Samier and Ismail (2015) and Rahul and Kandalkar (2018).

\subsection{Heterosis analysis}

Heterosis is interpreted by the effects of allelic and non-allelic interactions. In India, efforts on hybrid wheat were 
initiated in sixties following cytoplasmic male sterility (CMS) and chemical hybridizing agent (CHA) approach in 1995. However no significant results were obtained. It is well known that heterozygous genotypes are more adapted and stable under a variety of environments. Wheat hybrids are found to be stable for their performance in different environments. However, many wheat breeders address heterosis in their work. But production of enough hybrid grains for commercial use is still a dream.

Table (4): Estimates of general (of the parents) and specific (of crosses) combining ability effects for the studied traits.

\begin{tabular}{|c|c|c|c|c|c|c|c|}
\hline $\mathrm{F}_{\text {Genotypes }}$ Traits & $\begin{array}{l}\text { Days to } 50 \% \\
\text { blooming }\end{array}$ & $\begin{array}{l}\text { Plant } \\
\text { height }\end{array}$ & $\begin{array}{l}\text { Number of } \\
\text { spikes/plants }\end{array}$ & $\begin{array}{l}\text { Spike } \\
\text { length }\end{array}$ & $\begin{array}{l}\text { Number of } \\
\text { grains/ spike }\end{array}$ & $\begin{array}{l}\text { 1000-grain } \\
\text { weight }\end{array}$ & $\begin{array}{l}\text { Grain } \\
\text { yield/plant }\end{array}$ \\
\hline Sids 1 ( P1 ) & $-0.540 * *$ & $1.093 * *$ & $0.579 * *$ & $-0.457 * *$ & $-2.276^{* *}$ & $-1.28 * *$ & $-1.938^{* * *}$ \\
\hline Sids 12( P2 ) & $0.307 * *$ & $-1.596^{*}$ & $-0.447 *$ & -0.071 & $0.831 * *$ & -0.124 & $0.225 * *$ \\
\hline Sids 13 ( P3 ) & 0.737 ** & $2.315^{* * *}$ & $0.668 * *$ & $0.457 * *$ & $0.993 * *$ & 1.41 ** & $0.965 * *$ \\
\hline Shandaweel 1( P4 ) & $-0.119^{*}$ & $-1.793 * *$ & $-0.510^{*}$ & $-0.888 * *$ & $-1.322 * *$ & $-.1 .742 * *$ & $-0.234^{* *}$ \\
\hline Giza 171( P5 ) & $-0.684 * *$ & $-2.081 * *$ & $0.425 * *$ & $0.662 * *$ & $4.048 * *$ & $3.094 * *$ & $1.110^{* * *}$ \\
\hline Gemmeiza ( P6 ) & $-0.453^{*}$ & $3.152 * *$ & $-0.28 * *$ & 0.032 & $-3.022 * *$ & $-1.784^{*}$ & $-0.386^{* *}$ \\
\hline Sakha 93 ( P7 ) & 0.752 *** & $-1.01 * *$ & $0.41 * *$ & $0.329 * *$ & $0.748 * *$ & $1.542 * *$ & 0.258 ** \\
\hline S.E gi & 0.060 & 0.065 & 0.048 & 0.041 & 0.079 & 0.075 & 0.069 \\
\hline $\mathrm{P} 1 \times \mathrm{P} 2$ & $-1.384 * *$ & $2.387 * *$ & $0.386 * *$ & $0.743 * *$ & -0.650 & 0.284 & 0.495 \\
\hline $\mathrm{P} 1 \times \mathrm{P} 3$ & $-0.447 *$ & $2.239 * *$ & $1.605 * *$ & -0.038 & $2.912 * *$ & $0.677^{*}$ & $2.952 * *$ \\
\hline $\mathrm{P} 1 \times \mathrm{P} 4$ & 0.209 & $1.461 * *$ & $0.749 * *$ & $1.184 * *$ & -0.435 & $1.884 * *$ & $0.654^{*}$ \\
\hline $\mathrm{P} 1 \times \mathrm{P} 5$ & -0.052 & $1.409 * *$ & $0.497 * *$ & 0.110 & 0.158 & $1.699 * *$ & $0.843^{* *}$ \\
\hline $\mathrm{P} 1 \times \mathrm{P} 6$ & $-1.523^{* *}$ & $-0.761 * *$ & 0.019 & $0.807 * *$ & $0.787^{*}$ & -0.123 & $0.572^{*}$ \\
\hline $\mathrm{P} 1 \times \mathrm{P} 7$ & 1.571 *** & 1.469 ** & $-0.310^{*}$ & 0.936 ** & $4.876^{* *}$ & $2.918 * *$ & 1.095 ** \\
\hline $\mathrm{P} 2 \times \mathrm{P} 3$ & $1.172 * *$ & $1.328 * *$ & $0.397 * *$ & $0.276^{*}$ & 0.576 & $1.114 * *$ & 0.385 \\
\hline $\mathrm{P} 2 \times \mathrm{P} 4$ & $-0.906^{* *}$ & 0.183 & $-0.592 * *$ & $0.671 * *$ & $2.763 * *$ & $1.355^{* *}$ & $1.654 * *$ \\
\hline $\mathrm{P} 2 \times \mathrm{P} 5$ & -0.006 & $3.065^{* *}$ & $-1.110 * *$ & $0.724 * *$ & $3.889 * *$ & $1.969 * *$ & $1.110^{* *}$ \\
\hline $\mathrm{P} 2 \times \mathrm{P} 6$ & 0.229 & $4.294 * *$ & $2.379 * *$ & $0.754 * *$ & $5.485 * *$ & $1.214 * *$ & $-1.294 * *$ \\
\hline $\mathrm{P} 2 \times \mathrm{P} 7$ & $-1.243^{*}$ & $1.257 * *$ & $2.582 * *$ & $0.517 * *$ & $-0.759 *$ & $1.421 * *$ & $-4.661 * *$ \\
\hline $\mathrm{P} 3 \times \mathrm{P} 4$ & 0.387 & $7.835^{* *}$ & $0.727 * *$ & $1.284 * *$ & $1.791 * *$ & $0.881 * *$ & \begin{tabular}{|l}
$0.678^{*}$ \\
\end{tabular} \\
\hline $\mathrm{P} 3 \times \mathrm{P} 5$ & \begin{tabular}{|l}
$0.586^{*}$ \\
\end{tabular} & $2.017 * *$ & $0.975 * *$ & $1.3494 * *$ & $1.384 * *$ & $1.562 * *$ & $1.000^{* *} *$ \\
\hline $\mathrm{P} 3 \times \mathrm{P} 6$ & 1.189 ** & $2.913^{* *}$ & $0.464 * *$ & $0.207^{*}$ & $-2.387 * *$ & $1.173^{* *} *$ & $1.096^{* *} *$ \\
\hline $\mathrm{P} 3 \times \mathrm{P} 7$ & $-0.950 * *$ & $0.643^{*}$ & $-0.799 * *$ & $0.803 * *$ & $3.969 * *$ & $1.847 * *$ & $-1.015^{* *}$ \\
\hline $\mathrm{P} 4 \times \mathrm{P} 5$ & -0.081 & $1.472 * *$ & -0.047 & $0.832 * *$ & $1.704 * *$ & $1.103 * *$ & 0.402 \\
\hline $\mathrm{P} 4 \times \mathrm{P} 6$ & $-1.579 * *$ & $2.002 * *$ & $1.108 * *$ & $1.428 * *$ & $2.633 * *$ & $2.014 * *$ & -0.002 \\
\hline $\mathrm{P} 4 \times \mathrm{P} 7$ & $0.483^{*}$ & $-0.602 *$ & $0.645 * *$ & $0.658 * *$ & -0.478 & 0.521 & $1.654 * *$ \\
\hline $\mathrm{P} 5 \times \mathrm{P} 6$ & $0.650^{* * *}$ & $-1.217 * *$ & $1.590 * *$ & $0.621 * *$ & $5.993 * *$ & $3.329 * *$ & $2.054 * *$ \\
\hline $\mathrm{P} 5 \times \mathrm{P} 7$ & 0.251 & 1.746 *** & 0.760 *** & $0.518 * *$ & $4.015^{* *}$ & 0.303 & $2.343 * *$ \\
\hline $\mathrm{P} 6 \times \mathrm{P} 7$ & $0.513^{*}$ & $2.543 * *$ & $0.616^{* *}$ & $0.547 * *$ & $-1.356^{*}$ & $1.781 * *$ & $4.606^{* * *}$ \\
\hline S.E sij & 0.211 & 0.254 & 0.133 & 0.100 & 0.368 & 0.330 & 0.275 \\
\hline
\end{tabular}

Mid and best parent heterosis are available. However, the real feasibility of hybrids depends on the heterotic advantage over the best parent or the best common grown cultivar.The results of heterosis are presented in Tables (5) and (6). Eighteen and nine hybrids showed significant heterosis in $\mathrm{PH}$ over the mid and better parent, respectively. Five of them showed significant sca effects in PH. For days to $50 \%$ blooming, 13 hybrids gave negative favorable significant heteroses, only five of them depend on negative SCA. 
Table (5): Heterosis in percentage of mid-parents (M.P) and better parent (B.P) in the $\mathrm{F}_{1}$ crosses for Plant height, 50\% blooming (day), number of spikes/plant and spike length.

\begin{tabular}{|c|c|c|c|c|c|c|c|c|}
\hline \multirow{2}{*}{ Crosses $\quad$ Traits } & \multicolumn{2}{|c|}{ Days to $50 \%$ blooming } & \multicolumn{2}{|c|}{ Plant height } & \multicolumn{2}{|c|}{ Number of spikes/plant } & \multicolumn{2}{|c|}{ Spike length } \\
\hline & M.P & B.P & M.P & B.P & M.P & B.P & M.P & B.P \\
\hline $\mathrm{P} 1 \times \mathrm{P} 2$ & $-2.73 * *$ & $-3.95 * *$ & $7.70 * *$ & $13.73 * *$ & $17.78 * *$ & 6.30 & $10.75 * *$ & $17.01 * *$ \\
\hline $\mathrm{P} 1 \times \mathrm{P} 3$ & $-1.16^{*}$ & $-2.51 *$ & $8.92 * *$ & 2.94 & $23.96^{* *}$ & 23.78 & $15.45^{* *}$ & 15.16 \\
\hline $\mathrm{P} 1 \times \mathrm{P} 4$ & -0.25 & 0.08 & 6.72 & 3.04 & $17.35^{*}$ & 8.55 & $12.13 * *$ & $20.92 * *$ \\
\hline $\mathrm{P} 1 \times \mathrm{P} 5$ & -0.42 & 0.08 & 5.40 & 4.43 & $15.32 *$ & 7.27 & $16.57 * *$ & $22.79 * *$ \\
\hline $\mathrm{P} 1 \times \mathrm{P} 6$ & $-2.30 * *$ & -2.40 & 3.75 & 1.90 & $19.81^{* *}$ & 4.80 & $22.79 * *$ & $19.53 *$ \\
\hline $\mathrm{P} 1 \times \mathrm{P} 7$ & $1.18 *$ & -0.28 & 5.30 & 3.43 & 9.97* & 7.50 & $22.90 * *$ & $19.10 *$ \\
\hline $\mathrm{P} 2 \times \mathrm{P} 3$ & $0.55 *$ & 0.44 & $9.45 * *$ & $7.31 *$ & $18.81^{* *}$ & 7.29 & $17.36^{* *}$ & 13.45 \\
\hline $\mathrm{P} 2 \times \mathrm{P} 4$ & $-1.97 * *$ & $-2.87 * *$ & $6.73 * *$ & $6.46^{*}$ & 9.70 & 6.79 & $23.38 * *$ & $20.04 *$ \\
\hline $\mathrm{P} 2 \times \mathrm{P} 5$ & $-0.59 * *$ & $-2.46^{*}$ & $8.38 * *$ & $3.47 *$ & 4.19 & 1.74 & $20.69 * *$ & 20.50 \\
\hline $\mathrm{P} 2 \times \mathrm{P} 6$ & $-0.76^{*}$ & $-1.91 *$ & $9.7 * *$ & $3.88 *$ & 27.68 ** & $42.59 * *$ & $21.53 * *$ & $20.88 *$ \\
\hline $\mathrm{P} 2 \times \mathrm{P} 7$ & $-2.21 * *$ & $-2.41 *$ & $6.38 * *$ & $4.27 *$ & $38.10^{* *}$ & $27.25^{*}$ & $18.87 * *$ & 18.87 \\
\hline $\mathrm{P} 3 \times \mathrm{P} 4$ & $-1.26^{*}$ & $-2.27 *$ & $6.39 * *$ & $13.85^{* *}$ & $18.03^{* * *}$ & 9.24 & $29.21 * *$ & $21.65 *$ \\
\hline $\mathrm{P} 3 \times \mathrm{P} 5$ & $0.70 *$ & $-1.27 *$ & 8.58 & 1.73 & 20.11 ** & 11.80 & $26.75^{* *}$ & $22.35 *$ \\
\hline $\mathrm{P} 3 \times \mathrm{P} 6$ & $-0.81 *$ & $-2.06^{*}$ & $9.62 * *$ & 1.89 & $24.26^{* *}$ & 9.02 & $18.26^{* *}$ & 13.68 \\
\hline $\mathrm{P} 3 \times \mathrm{P} 7$ & $-1.88 * *$ & $-1.99 *$ & $7.08 * *$ & 2.95 & 7.07 & 4.73 & $14.63 * *$ & 11.70 \\
\hline $\mathrm{P} 4 \times \mathrm{P} 5$ & 0.53 & -0.45 & $6.72 * *$ & 2.12 & 11.14 & 10.46 & $26.22 * *$ & $22.16^{*}$ \\
\hline $\mathrm{P} 4 \times \mathrm{P} 6$ & $0.83 *$ & 0.57 & $7.36 * *$ & 1.89 & $21.05 * *$ & $33.30 * *$ & $29.84 * *$ & $26.33 *$ \\
\hline $\mathrm{P} 4 \times \mathrm{P} 7$ & $-1.69 *$ & $-2.79 *$ & $4.37 * *$ & 2.54 & $18.03^{* *}$ & 11.54 & $23.83 * *$ & $20.48 *$ \\
\hline $\mathrm{P} 5 \times \mathrm{P} 6$ & $-6.59 * *$ & $-10.38 * *$ & $3.05 * *$ & 2.18 & $15.41^{*}$ & 26.67 & $11.35^{* *}$ & $20.50 * *$ \\
\hline $\mathrm{P} 5 \times \mathrm{P} 7$ & \begin{tabular}{|l|}
0.32 \\
\end{tabular} & $-1.75^{*}$ & $5.59 * *$ & $8.55 * *$ & $19.0^{* * *}$ & 13.11 & $19.69 * *$ & 19.50 \\
\hline $\mathrm{P} 6 \times \mathrm{P} 7$ & $-1.60 *$ & $-1.75^{*}$ & $6.43 * *$ & $2.74 * *$ & $26.76^{* *}$ & $31.11 * *$ & $20.52 * *$ & 19.87 \\
\hline
\end{tabular}

* Significant at $5 \%$ level of significance, $* *$ significant at $1 \%$ level of significance.

Table (6): Heterosis in percentage of mid-parents (M.P) and better parent (B.P) in the $F_{1}$ crosses for number of grains /spikes, 1000grain weight and grain yield/plant (gm).

\begin{tabular}{|c|c|c|c|c|c|c|}
\hline \multirow{2}{*}{${ }_{\text {Crosses }}$ Traits } & \multicolumn{2}{|c|}{ Number of grains /spike } & \multicolumn{2}{|c|}{ 1000-grain weight } & \multicolumn{2}{|c|}{ Grain yield/plant } \\
\hline & M.P & B.P & M.P & B.P & M.P & B.P \\
\hline $\mathrm{P} 1 \times \mathrm{P} 2$ & $5.31 *$ & 2.19 & 8.55 & 6.03 & $12.60 * *$ & $11.29 *$ \\
\hline $\mathrm{P} 1 \times \mathrm{P} 3$ & $8.86 * *$ & 4.87 & $6.64 * *$ & $10.09 * *$ & $11.72 * *$ & $9.38 *$ \\
\hline $\mathrm{P} 1 \times \mathrm{P} 4$ & 4.60 & 3.40 & $12.30 * *$ & $9.69 *$ & $11.38 * *$ & $10.21 *$ \\
\hline $\mathrm{P} 1 \times \mathrm{P} 5$ & 13.26 & 2.95 & $12.41 * *$ & 4.45 & $14.20 * *$ & $12.64 *$ \\
\hline $\mathrm{P} 1 \times \mathrm{P} 6$ & $6.93 * *$ & $11.26^{* *}$ & $9.24 * *$ & $6.55^{*}$ & $13.22 * *$ & $9.16^{*}$ \\
\hline $\mathrm{P} 1 \times \mathrm{P} 7$ & $11.82 * *$ & $5.89 *$ & $13.90 * *$ & $8.19 *$ & $21.83 * *$ & $13.61 *$ \\
\hline $\mathrm{P} 2 \times \mathrm{P} 3$ & $5.83^{* *}$ & $9.02^{* *}$ & $10.80 * *$ & $7.53 *$ & $11.08 * *$ & $9.97 *$ \\
\hline $\mathrm{P} 2 \times \mathrm{P} 4$ & $9.68 * *$ & $7.64 *$ & $11.07 * *$ & $11.07^{*}$ & $10.23 * *$ & $17.10 * *$ \\
\hline $\mathrm{P} 2 \times \mathrm{P} 5$ & 15.08 & 5.66 & $12.85 * *$ & $7.23 *$ & $10.28 * *$ & $14.40 *$ \\
\hline $\mathrm{P} 2 \times \mathrm{P} 6$ & $5.61^{*}$ & $10.80 * *$ & $12.22 * *$ & 6.97 & $8.39 * *$ & 5.91 \\
\hline $\mathrm{P} 2 \times \mathrm{P} 7$ & $5.68^{*}$ & 3.06 & $11.27 * *$ & 8.13 & $21.20 * *$ & $30.29 * *$ \\
\hline $\mathrm{P} 3 \times \mathrm{P} 4$ & $7.44 * *$ & 4.66 & $10.27 * *$ & 7.25 & $10.32 * *$ & 9.10 \\
\hline $\mathrm{P} 3 \times \mathrm{P} 5$ & $10.52^{* * *}$ & 0.78 & $12.50 * *$ & 3.93 & $13.70^{* *}$ & $9.83 *$ \\
\hline $\mathrm{P} 3 \times \mathrm{P} 6$ & 3.00 & 1.19 & $12.39 * *$ & $10.09^{*}$ & $14.09 * *$ & $12.54 *$ \\
\hline $\mathrm{P} 3 \times \mathrm{P} 7$ & 10.47 ** & $8.53 *$ & $12.41 * *$ & 6.36 & $12.47 * *$ & 7.00 \\
\hline $\mathrm{P} 4 \times \mathrm{P} 5$ & $11.16^{\text {** }}$ & 3.87 & $11.28 * *$ & 5.73 & $12.94 * *$ & $10.29 *$ \\
\hline $\mathrm{P} 4 \times \mathrm{P} 6$ & $9.26 * *$ & 4.61 & $6.25 * *$ & 8.91 ** & $11.40 * *$ & 8.77 \\
\hline $\mathrm{P} 4 \times \mathrm{P} 7$ & $5.09 *$ & 0.63 & $4.60^{*}$ & 6.50 & $14.26 * *$ & $17.21 * *$ \\
\hline P5×P6 & $17.42^{* *}$ & 5.38 & $17.44 * *$ & 6.65 & $21.17 * *$ & $15.56^{*}$ \\
\hline $\mathrm{P} 5 \times \mathrm{P} 7$ & $14.49^{* *}$ & 2.75 & $9.76^{* *}$ & $7.23 *$ & $29.02 * *$ & $18.82 *$ \\
\hline $\mathrm{P} 6 \times \mathrm{P} 7$ & 2.75 & 2.75 & 3.69 & 5.48 & 3.73 & 7.20 \\
\hline
\end{tabular}

* Significant at $5 \%$ level of significance, ** significant at $1 \%$ level of significance.

Respect to the better parent heterosis in the other traits, results showed that three out of four hybrids for NS/P, nine out of 13 for SL, five out of six for NG/S, five out of ten for GW and five out of 16 hybris for GY/P showed significant better parent heterosis based on SCA. These results indicate that heterotic 
effects in these materials depend on both additive and non-additive gene effects. These results are supported with the findings of Ashoush et al. (2001), Jahanzeb and Ihsan (2004), El-Sayed and
Moshref (2005), Abdel-Moneam (2009), Bertan et al. (2009), Peng et al. (2009), Kundan et al. (2010), Ahmad (2010), Zaazaa et al. (2012), Samier and Ismail (2015) and Rahul and Kandalkar (2018).

Table (7): Correlation among studied characters for genotypes.

\begin{tabular}{|l|c|c|c|c|c|c|c|}
\hline Characters & $\begin{array}{c}\text { Days to } \\
50 \% \text { blooming }\end{array}$ & $\begin{array}{c}\text { Plant } \\
\text { height }\end{array}$ & $\begin{array}{c}\text { Number of } \\
\text { spikes /plant }\end{array}$ & Spike length & $\begin{array}{c}\text { Grain } \\
\text { weight/spike }\end{array}$ & $\begin{array}{c}1000-\text { grain } \\
\text { weight }\end{array}$ & $\begin{array}{c}\text { Grain } \\
\text { yield/plant }\end{array}$ \\
\hline Days to 50\% blooming & -- & $-0.355^{* *}$ & -0.141 & -0.003 & -0.003 & $-0.376^{* *}$ & $-0.328^{* *}$ \\
\hline Plant height & & -- & -0.029 & 0.162 & -0.022 & $0.278^{*}$ & $-0.373^{* *}$ \\
\hline Number of spikes /plant & & & -- & 0.120 & $-0.339^{* *}$ & $0.291^{*}$ & $0.356^{* *}$ \\
\hline Spike length & & & -- & $0.421^{* *}$ & 0.231 & $0.369^{* *}$ \\
\hline Grain weight / spike & & & & & -- & $-0.367^{* *}$ & $0.388^{* *}$ \\
\hline 1000-grain weight & & & & & -- & $-0.329^{* *}$ \\
\hline Grain yield/plant & & & & & -- \\
\hline
\end{tabular}

\subsection{Phenotypic correlation}

The correlation values between each pairs of studied traits were shown in Table (7). Grain yield/plant showed significant $(\mathrm{p} \leq 0.01)$ correlation with $\mathrm{NS} / \mathrm{P}$, SL and NG/S, while it gave negative correlation with $\mathrm{GW}, \mathrm{PH}$, and days to $50 \%$ blooming. Therefore, selection for NS/P, SL and NG/S in these materials may result in high yielding early lines of short stature, and selection for SL could increase NG/S. These results are supported with the findings of Ashoush et al. (2001), El-Sayed and Moshref (2005), Abdel-Moneam (2009), Peng et al. (2009), Kundan et al. (2010), Ahmad (2010), Farooq et al. (2010), Khaled and Abd El-dayem (2014), Samier and Ismail (2015) and Rahul and Kandalkar (2018).

\section{References}

Abd El-Mageed, S. A. (1995),
Inheritance of yield, yield components and some morphological characters in spring wheat crosses, Ph.D. Thesis, Faculty of Agriculture, Minia University, Minia, Egypt.

Abdel-Moneam, M. A. (2009), "Heterosis in some crosses of bread wheat under irrigation and drought conditions", Pakistan Journal of Biological Sciences, Vol. 12, pp. 486-491.

Abdel-Nour Nadya, A. R. and Hassan Manal, A. (2009), "Determination of gene effects and variance in three bread wheat crosses for low water (Drought)", Egyptian Journal of Plant Breeding, Vol. 13, pp. 235249.

Afiah, S. A. N., Mohamed, N. A. and Salem, M. M. (2000), "Statistical genetic parameters, heritability and graphical analysis in $8 \times 8$ wheat diallel crosses under saline 
conditions", Annals of Agricultural Science, Vol. 45 No. 1, pp. 257-280.

Ahmad, M. S. H. (2010), "Combining ability and heterosis estimates for agronomic traits in bread wheat using half diallel analysis", Journal of Plant Production, Vol. 1 No. 11, pp. 1441-1450.

Ahmadi, J., Zali, A. A., Yazdi, S. B., Talaie A., Ghannadha, M. R. and Saeidi, A. (2003), "A study of combining ability and gene effect in bread wheat under drought stress condition by diallel method", Iranian Journal of Agriculture Science, Vol. 34 No. 1, pp. 1-8.

Ashoush, H. A., Hamada, A. A. and Darwish, I. H. (2001), "Heterosis and combining ability in $F_{1}$ and $F_{2}$ diallel crosses of wheat (Triticum aestivum L. em. Thell)", Journal of Plant Production, Vol. 26 No. 5, pp. 2579-2592.

Bertan, I., Carvalho, F., Oliveira, A., Benin, G., Vieira, E. and Valério, I. (2009), "Morphological, pedigree, and molecular distances and their association with hybrid wheat performance", Pesquisa Agropecuária Brasileira, Vol. 44 No. 2, pp. 155-163.

Darwish, I. H. (1992), Breeding studies on wheat, M.Sc Thesis, Faculty of Agriculture, Menufiya University, Egypt.

EI-Sayed, E. A .M. (2004), "Adiallel cross analysis for some quantitative characters in bread wheat (Triticum aestivum L.)", Egyptian Journal of Agricultural Research, Vol. 82 No. 4, pp.1665-1679.

El- Shal, M. H., Habib, E. M. and Arab, S. A. (2014), "Estimation of combining ability and gene action in some Egyptian wheat landraces", Egyptian Journal of Agronomy, Vol. 36 No. 2, pp. 205-217.

El-Awady Wafaa, A. (2011), "Analysis of yield and its components using five parameters for three bread wheat crosses", Egyptian Journal of Agricultural Research, Vol. 89 No. 3, pp. 993-1003.

El-Beially, E. M. A. and El-Sayed, E. A. M. ( 2002), "Heterosis and combining ability for some bread wheat crosses", Journal of Plant Production, Vol. 27, pp. 57355744.

El-Borhamy, H. S. A. (2004), "Genetic analysis of some drought and yield related characters in spring wheat varieties (Triticum aestivum $\mathrm{L}$. em. Thell)", Journal of Plant Production, Vol. 29 No. 7, pp. 3719-3729.

El-Hennawy, M. A. (1992), "Inheritance of grain yield and other agronomic characters in two wheat crosses", Al-Azhar Journal of Agricultural Research, Vol. 15, pp.b57-68.

El-Sayed, E. M. A. and Moshref, M. K. (2005), "Breeding for yield, yield components and some agronomic 
characters in bread wheat", Egyptian Journal of Agricultural Research, Vol. 83 (2): 665-63.

Farooq, J., Khaliq, I., Khan, A. S. and Pervez, M. A. (2010), "Studying the genetic mechanism of some yield contributing traits in wheat (Triticum aestivum)", International Journal of Agriculture \& Biology, Vol. 12 No 2, pp. 1560-8530.

Fonseca, S. and Patterson, F. L. (1968), "Hybrid vigor in seven parental diallel cross in common wheat (Ttiticum aestivum L.)", Crop Science, Vol. 8, pp. 85-88.

Griffing, B. (1956), "Concept of general and specific combining ability in relation to diallel crossing", Assiut Journal of Biological Science, Vol. 9, pp. 463-493.

Hamada, A. A. and El-Beially, E. M. A. (2003), "Assessment of some breeding parameters for yield and its attributes in bread wheat (Triticum aestivum L.)", Egyptian Journal of Applied Science, Vol. 18 No. 12, pp. 159-176.

Hayman, B. I. (1954a), "The theory of analysis of diallel crosses II", Genetics, Vol. 43, pp. 789-809.

Hayman, B. I. (1954b), "The analysis of variance of diallel tables", Biometrics, Vol. 10, pp. 235-244.

Hendawy, H. I. (1990), Breeding for yield and its components in wheat, M.Sc. Thesis, Faculty of
Agriculture, Menoufiya University, Egypt.

Jahanzeb, F. and Ihsan, K. (2004), "Estimation of heterosis and heterobeltiosis of some quantitative characters in bread wheat crosses", Asian Journal of Plant Science, Vol. 3 No. 4, pp. 508-511.

Jinks, J. L. (1954), "The analysis of continuous variation in a diallel cross of Nicotinia rustica varieties", Genetics, Vol. 39, pp. 767-788.

Khaled, M. A. and Abd El-dayem, S. M. (2014), "Combining ability analysis for grain yield and its attributes in bread wheat under stress and normal irrigation conditions", Journal of Plant Production, Vol. 5 No. 2, pp. 255-266.

Khalifa, M. A., El-Morshidy, M. A., Hassaballa, E. A. and Ismail, A. A (1984), "Inheritance of some agronomic characters in wheat (Triticum aestivum L.)", Assiut Journal of Agriculture Science, Vol. 15 , pp. 217-233.

Kumar, B. and Gupta, B. B. (2010), "Combining ability in F1 hybrids of a diallel cross of bread wheat (Triticum aestivum L.)", Environment and Ecology, Vol. 28, pp. 494-498.

Kundan, K., Pandey, J. P., Marker, S. and Anurag, P. J. (2010), "Heterosis studies for improvement in yield potential of wheat (Triticum aestivum L.)", Genetic Engineering 
and Biotechnology Division, Vol. 9 No. 10, pp. 6394-6403.

Madic, M., Paunovic, A., Durovic, D., Kraljvic-Balalic, M. and knezevic, D. (2005), "The analysis of gene effect in the inheritance of kernel number per spike in barley hybrid", Genetika, Vol. 37, pp. 261-269.

Mahmoud, K. A. H. (1999), Genetic studies on some yield traits of durum wheat, M.Sc. Thesis, Faculty of Agriculture, Assuit University, Assuit, Egypt.

Megahed Eman, M. A., Ramadan, I. E., Awaad, H. A., Swelam, A. A. and Ali, M. M. A. (2014), "Heterobeltiosis for morphophysiological and yield characters of bread wheat under different levels of nitrogen", Zagazig Journal of Agricultural Research, Vol. 41 No. 2, pp. 219-230.

Motawea, M. H. (2006), "Inheritance of earliness, yield and yield components in wheat (Triticum aestivum L.)", Assiut Journal of Agriculture Science, Vol. 37 No. 2, pp. 35-52.

Moussa, A. M. (2010), "Estimation of epistasis, additive and dominance variation in certain bread wheat (Triticum aestivum, L) crosses", Journal of Plant Production , Mansoura University, Vol. 1 No. 12, pp. 1707-1719.

Nazir, S., Khan, A. S. and Ali, Z. (2005), "Combining ability analysis for yield and yield contributing traits in bread wheat", Journal of Agriculture Science,6 (2): 137-154.

Padhar, P. R., Madaria, R. B., Vachhani, J. H. and Dobariya, L. (2010), "Combining ability analysis of grain yield and its contributing characters in bread wheat (Triticum aestivum L.) under late sown condition", International Journal of Agricultural Sciences, Vol. 6 No.1, pp. 267-272.

Peng, Z., Ru, P. H., Fu, N. Z., Hu, L. L., Jun, W. and Xin, S. Q. (2009), "Heterosis and combining ability of major agronomic traits in crosses between ear-branched wheat and common wheat", Journal of Triticeae Crops, Vol. 29, pp. 212216.

Rahul, S. R. and Kandalkar, V. S. (2018), "Combining ability and heterosis for grain yield and its attributing traits in bread wheat (Triticum aestivum L.)", Journal of Pharmacognosy and Phytochemistry, Vol. 7 No. 2, 113119.

Samier, K. and Ismail, A. (2015), "Heterosis and combining ability analysis for yield and its components in bread wheat (Triticum aestivum L.)", International Journal of Current Microbiology and Applied Sciences, Vol. 4 No. 8, pp. 1-9.

Sener, O. (2009), "Identification of breeding potential for grain yield and its component traits of common 
wheat varieties in the East Tolba, A. M. (2000), "Diallel analysis of Mediterranean", Agrociencia Montecillo, Vol. 43, pp. 707-716.

Snedecor, G. W. and Cochran, W. G. (1982), Statistical methods, $7^{\text {th }}$ edition, Iowa State University Press, Ames, USA.

Subhashchandra, B., Lohithaswa, H. C., Desai, S. A., Hanchinal, R. R., Kalappanavar, I. K., Math, K. K. and Salimath, P. M. (2009), "Assessment of genetic variability and relationship between genetic diversity and transgressive segregation in tetraploid wheat", Karnataka Journal of Agricultural Sciences, 22: 36-38. yield and its attributes in bread wheat (Triticum aestivum L.)", Egyptian Journal of Plant Breeding, Vol. 4, pp. 71-87.

Zaazaa, E. I., Hager, M. A. and ElHashash, E. F. (2012), "Genetical analysis of some quantitative traits in wheat using six parameters genetic model", American-Eurasian Journal of Agriculture \& Environmental Science, Vol. 12 No. 4, pp. 456-462. 